\title{
The early spectral evolution of SN 2004dt
}

\author{
G. Altavilla ${ }^{1,2}$, M. Stehle ${ }^{3}$, P. Ruiz-Lapuente ${ }^{1,3}$, P. Mazzali ${ }^{3,4}$, G. Pignata ${ }^{5}$, A. Balastegui ${ }^{1}$, S. Benetti ${ }^{6}$, G. Blanc ${ }^{6, \star}$, \\ R. Canal ${ }^{1}$, N. Elias-Rosa ${ }^{6,7, \star \star}$, A. Goobar ${ }^{8}$, A. Harutyunyan ${ }^{6}$, A. Pastorello ${ }^{3, \star \star \star}$, F. Patat ${ }^{9}$, J. Rich $^{10}$, M. Salvo ${ }^{11}$, \\ B. P. Schmidt ${ }^{11}$, V. Stanishev ${ }^{8}$, S. Taubenberger ${ }^{3}$, M. Turatto ${ }^{6}$, and W. Hillebrandt ${ }^{3}$
}

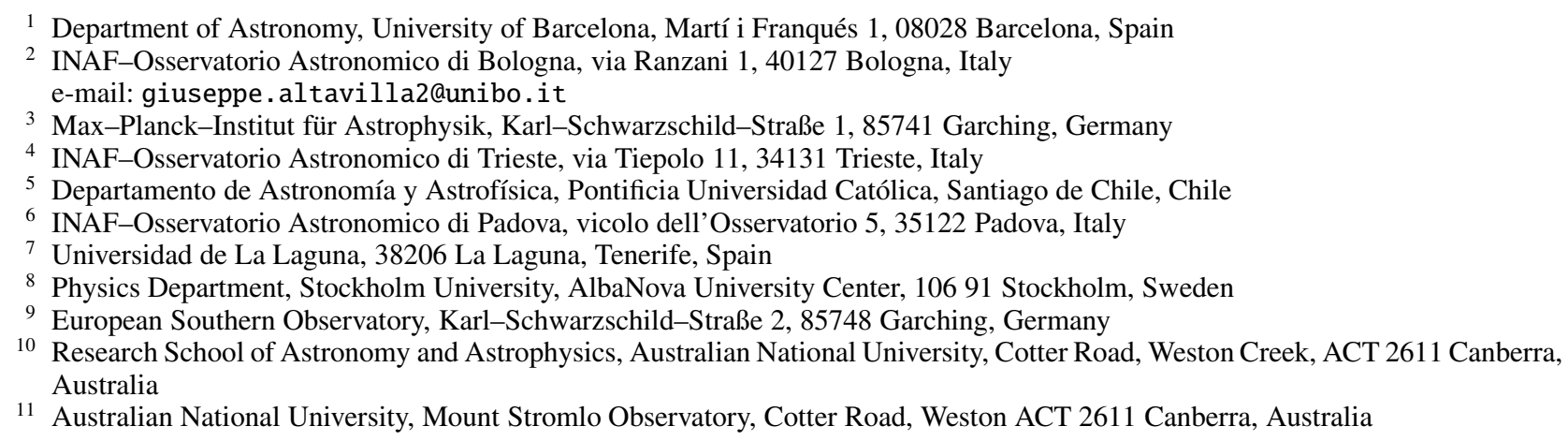

Received 16 March 2007 / Accepted 8 August 2007

\section{ABSTRACT}

\begin{abstract}
Aims. We study the optical spectroscopic properties of Type Ia Supernova (SN Ia) 2004dt, focusing our attention on the early epochs. Methods. Observation triggered soon after the SN 2004dt discovery allowed us to obtain a spectrophotometric coverage from day -10 to almost one year ( $\sim 353$ days) after the $B$ band maximum. Observations carried out on an almost daily basis allowed us a good sampling of the fast spectroscopic evolution of SN 2004dt in the early stages. To obtain this result, low-resolution, long-slit spectroscopy was obtained using a number of facilities.

Results. This supernova, which in some absorption lines of its early spectra showed the highest degree of polarization ever measured in any SN Ia, has a complex velocity structure in the outer layers of its ejecta. Unburnt oxygen is present, moving at velocities as high as $\sim 16700 \mathrm{~km} \mathrm{~s}^{-1}$, with some intermediate-mass elements ( $\left.\mathrm{Mg}, \mathrm{Si}, \mathrm{Ca}\right)$ moving equally fast. Modeling of the spectra based on standard density profiles of the ejecta fails to reproduce the observed features, whereas enhancing the density of outer layers significantly improves the fit. Our analysis indicates the presence of clumps of high-velocity, intermediate-mass elements in the outermost layers, which is also suggested by the spectropolarimetric data.
\end{abstract}

Key words. stars: supernovae: general - stars: supernovae: individual: SN 2004dt - methods: observational techniques: spectroscopic

\section{Introduction}

Type Ia supernovae (SNe Ia) are thermonuclear explosions of white dwarfs (WDs) mostly originating from ignition of carbon close to the center of the star when it approaches the Chandrasekhar mass, due to accretion of matter from a close binary companion (see Hillebrandt \& Niemeyer 2000 for a review). The nature of the binary system and the mechanism of the explosion are still unsolved problems. Interest in solving these questions has strongly increased with the use of SNe Ia as distance indicators in cosmology, in particular by their role in the discovery of the acceleration of the expansion of the Universe (Riess et al. 1998; Perlmutter et al. 1999). The nature of the companion (whether it is another WD - the double-degenerate

^ Now at APC, Université Paris 7, 10 rue Alice Domon et Léonie Duquet, 75205 Paris Cedex 13, France.

$\star \star$ Now at Max-Planck-Institut für Astrophysik, Karl-Schwarzschild-Straße 1, 85741 Garching, Germany.

$\star \star \star$ Now at the Astrophysics Research Centre, School of Mathematics and Physics, Queen's University Belfast, BT7 1NN, Belfast, UK. or DD scenario - or a non-degenerate star in any evolutionary stage - the single - degenerate or SD scenario) is the object of different approaches: searches for DD systems with total mass exceeding the Chandrasekhar mass and able to merge in less than the Hubble time (Napiwotzki et al. 2004); searches for semidetached SD systems where a WD close to the Chandrasekhar mass is growing by accretion (Maxted et al. 2000); and finally searches for traces of hydrogen (whether deposited on the surface of the exploding WD, residing in an accretion disk, stripped of the companion, or present as circumstellar material) in early SNe Ia spectra (Lundqvist et al. 2003). A more direct approach, which is based on a possible identification of the surviving companion of the WD in the central regions of the remnants of the Galactic historical SNe Ia, would also support the SD scenario (Ruiz-Lapuente et al. 2004).

Concerning the explosion mechanism, there is general agreement that explosive thermonuclear burning starts near the center of the WD and first propagates subsonically outwards (deflagration). There is strong debate, though, on whether subsonic burning alone can produce both explosions with the typical SNe Ia 
energies and the chemical stratification revealed by the time sequence of SNe Ia spectra (avoiding, in particular, excessive mixing of unburnt carbon and oxygen within deep layers of the SN ejecta: see Reinecke et al. 2002; Gamezo et al. 2003). Transition to detonation (supersonic burning propagated by a shock wave) has been invoked when burning reaches layers with densities low enough to burn only partially when they detonate (delayed detonation). This takes place either in a spherical shell (Khokhlov 1991) or in a point (Livne 1999), but it has to be triggered artificially in current numerical simulations. Such detonations leave none or little of the carbon and oxygen unburnt. Recent 2D simulations have shown a detonation forming in the outer layers of an exploding WD when a hot plume rising from an off-center ignition spreads over the surface and sweeps the unburnt material that, when confined by the strong gravitational field, converges to a point (Plewa et al. 2004). Nevertheless, subsequent 3D simulations do not support this hypothesis, even if detonation, triggered by other mechanisms such as a pulsation or spontaneous detonation if the turbolent energy is high enough, is not completely ruled out (Röpke et al. 2006).

From the observational point of view, much can be learned about the explosion mechanism from the detailed structure of the layers that are just below the surface of the WD when it explodes. This material becomes rapidly transparent as the $\mathrm{SN}$ ejecta expand, and a series of early-time spectra is thus required for studying this phase properly. A recent analysis of the distribution of the abundances in a large sample of SNe Ia (Mazzali et al. 2007) suggests that the properties of most, if not all, SNe Ia can be explained by a single explosion scenario, the delayed detonation. Pre-maximum spectropolarimetry of $17 \mathrm{SNe}$ Ia analyzed by Wang et al. (2007) strongly corroborates the delayed detonation model.

Spectra of SN 2004dt taken about 10 days before maximum light show a high degree of polarization across some lines (Wang et al. 2006), with Si II displaying the strongest polarization $(\sim 2 \%)$ while $\mathrm{OI}$ is hardly polarized. The velocity structure is also complex (already noticed by Patat et al. 2004), with O I, Mg II, Si II, and Ca II moving at high velocities ${ }^{1}$ while S II and Si III appear significantly slower. This, together with the polarization data, suggests the presence of an almost spherical $\mathrm{O}$ layer with intrusions of intermediate-mass elements. While the finger-like structure is typically found in pure deflagration models of the explosions, Kasen \& Plewa (2005) interpret the presence of Ca II moving at high velocities as a signature of the deflagration products brought to the surface by the rising plume in the model of Plewa et al. (2004), which are compressed and accelerated by the SN ejecta after the detonation has been triggered.

${ }^{1}$ A distinction should be made between high-velocity features that persist through the optical maximum and the "high-velocity features" (HVFs), as the the Ca II IR triplet, commonly seen at very early epochs ( $\sim 10$ days before maximum light). The high velocities characterizing these features, much higher than the photospheric velocities, suggest that these lines (or a component of the photospheric line if not detached) originate above the photosphere and that they are a different phenomena from to the normal photospheric absorption features. As discussed by Tanaka et al. (2006), HVFs may be the result of density and/or abundance enhancements produced by the explosion itself or by the interaction of the SN with the circumstellar material (in this hypothesis high mass-loss rates are required for the progenitor) or with an accretion disk (the absence of hydrogen lines in the early spectra weaken this model, but see Gerardy et al. 2004 and Mazzali et al. 2005a). The Ca II IR HVF is nicely explained within the gravitationally confined detonation (GCD) model by Kasen \& Plewa (2005).
Table 1. Main parameters or SN 2004dt and its host galaxy.

\begin{tabular}{|c|c|}
\hline $\begin{array}{l}\text { Parent galaxy: } \\
\text { Galaxy type: }\end{array}$ & $\begin{array}{l}\text { NGC 799 } \\
\text { (R')SB(s)a * }\end{array}$ \\
\hline & $\begin{array}{l}\text { (R')SB(s)a } \mathrm{a}^{\star} \\
5015 \mathrm{rm}^{-1}{ }^{\star}\end{array}$ \\
\hline Heliocentric Radial Velocity: & $5915 \mathrm{~km} \mathrm{~s}^{-1^{\star}}$ \\
\hline Radial velocity (vvir): & $5846 \mathrm{~km} \mathrm{~s}^{-1^{+}}$ \\
\hline Redshift: & $0.01973^{\dagger}$ \\
\hline Kinematical distance modulus: & $34.61^{\bullet}$ \\
\hline $\mathrm{RA}_{\mathrm{SN}}(\mathrm{J} 2000):$ & $02^{\mathrm{h}} 02^{\mathrm{m}} 12^{\mathrm{s}} .77$ \\
\hline $\operatorname{Dec}_{S N}(J 2000):$ & $-00^{\circ} 05^{\circ} .51 " .5$ \\
\hline Offset from nucleus: & $7^{\mathrm{s} .0 \mathrm{E}, 10 " .} 5 \mathrm{~N}$ \\
\hline Discovery epoch: & $\mathrm{JD}=2453228.97$ (Aug. 11th) \\
\hline Date of $B$ maximum: & $\mathrm{JD}=2453240.3 \pm 0.5($ Aug. $22 \mathrm{nd})$ \\
\hline Observed magnitude at max: & $B=15.33(0.02)$ \\
\hline$\Delta m_{15}(B)_{\mathrm{obs}}:$ & $1.21(0.05)$ \\
\hline Stretch factor $(B)$ : & $0.91(0.01)$ \\
\hline Galactic Extinction: & $A_{B}=0.010 \mathrm{mag}^{\diamond} ; A_{B}=0.109 \mathrm{mag}^{\triangleright}$ \\
\hline$(B-V)_{\max }:$ & $-0.03 \pm 0.02^{\triangleleft}$ \\
\hline$E(B-V)_{\max }:$ & $0.04 \pm 0.04 \triangleleft$ \\
\hline$E(B-V)_{\text {tail }}:$ & $0.11 \pm 0.03^{\triangleleft}$ \\
\hline
\end{tabular}

* NED (Nasa/ipac Extragalactic Database). ${ }^{\ddagger}$ Leda (Lyon-Meudon Extragalactic Database), corrected for LG infall onto Virgo. ${ }^{\dagger}$ Theureau et al. (1998). Leda (Lyon-Meudon Extragalactic Database), from vvir and $H_{0}=70 \mathrm{~km} \mathrm{~s}^{-1} \mathrm{Mpc}^{-1} .{ }^{\circ}$ Burstein \& Heiles (1982). ${ }^{\triangleright}$ Schlegel et al. (1998) (we make use of this value). ${ }^{\triangleleft}$ Galactic extinction correction applied.

The expansion velocity of the strong Si II absorption, starting from $\sim 16000 \mathrm{~km} \mathrm{~s}^{-1}$ at about 10 days maximum light and decreasing fast with time, also suggests that SN2004dt is a high-velocity gradient (HVG) event (see Benetti et al. 2005), as confirmed by a preliminary photometric analysis, and similar to other SNe such as SN 2002bo (Benetti et al. 2004), its twin SN 2002dj (Pignata et al. 2005), SN 2002er (Pignata et al. 2004; Kotak et al. 2005), and SN 2005cf (Pastorello et al. 2007; Garavini et al. 2007), which have been extensively observed within the same scientific project: "The Physics of Type Ia Supernova Explosions, a European Research Training Network" (RTN), carried out by the European Supernova Collaboration (ESC, Benetti et al. 2004).

Here we present a series of optical spectra of SN 2004dt, from more than one week before maximum light to almost one year after, focusing our attention on spectra obtained at early epochs. This is a subsample of the whole data set collected for SN 2004dt on behalf of the RTN program. The complete data set includes optical and infared photometric data (UBVRI photometry, 58 nights, from -10 to +500 days since $B$ maximum; $J H K$ photometry, 18 nights, from -8 to 357 days since $B$ maximum); and optical and infrared spectroscopy (32 nights in the optical range, from -10 to 353 days and 5 nights in IR, from -5 to +16 days since $B$ maximum), which will be analyzed and discussed in a forthcoming paper.

We examine here the peculiarities of SN 2004dt as inferred from the shape and velocities of the spectral features before maximum and attempt to model the spectra from 1-D hydrodynamical models of the explosion. We find that the best fits are obtained when we artificially raise the density of the outermost layers, reinforcing the idea that both the velocity structure and the line polarization observed are due to clumps of intermediatemass elements on and above the "photosphere" of the SN.

\footnotetext{
${ }^{2}$ http://www.mpa-garching.mpg.de/ rtn/
} 


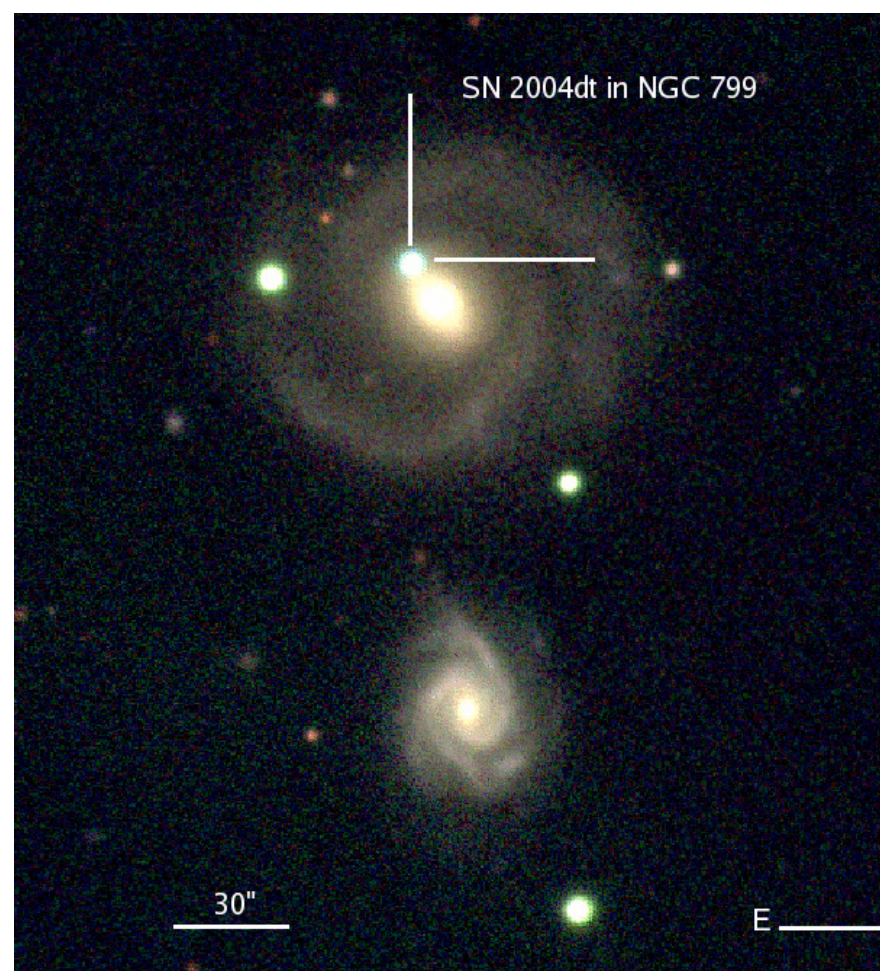

Fig. 1. SN 2004dt in NGC799. Color image obtained by combining $B$, $V, R$ images $(80,50,40 \mathrm{sec}$ exposure each) obtained on Aug. 19th with the $2.2 \mathrm{~m}+\mathrm{Cafos}$ at Calar Alto Observatory. The dashes indicate the position of the supernova.

\section{Observations and data reduction}

SN 2004dt (RA: $02^{\mathrm{h}} 02^{\mathrm{m}} 12^{\mathrm{s}} .77$, Dec: $-00^{\circ} 05^{\prime} 51^{\mathrm{s}} .5$, J2000) was discovered on August 11.48 UT by Moore \& Li (2004) on behalf of the LOSS/KAIT ${ }^{3}$ (Filippenko et al. 2001), in the spiral galaxy NGC 799, 7'.0 East and 10.'5 North from the galaxy nucleus (see Fig. 1 and Table 1 for the main parameters of SN 2004dt and its host galaxy).

Spectra of SN 2004dt were obtained immediately on August 12.72 UT at the ANU 2.3-m telescope (Salvo et al. 2004) and on August 13.17 UT at the Calar Alto 2.2-m telescope (Patat et al. 2004). On the basis of these spectra, the candidate was classified as a young Type Ia with some unusual features (Salvo et al. 2004; Patat et al. 2004). The proximity of the host galaxy $(z=0.01973$, Theureau et al. 1998), the early epoch of the discovery, the peculiar spectral features, the good position of the SN, easily observable from both the northern and southern observatories for a long period (the host galaxy had just appeared from behind the Sun), all this made SN 2004dt a good target for the ESC. For these reasons target-of-opportunity ( $\mathrm{ToO})$ observations were immediately triggered. Later on, observations were carried out either in service or in target-of-opportunity mode, using the configuration available at the requested time. Spectroscopy and imaging were carried out at several sites using a number of different instrumental configurations. Table 2 contains the log of the spectroscopic observations.

\footnotetext{
${ }^{3}$ Lick Observatory Supernova Search with the Katzman Automatic Imaging Telescope, http://astro.berkeley. edu/ bait/ kait.html supernova search.
}

Data reduction was performed using standard $\mathrm{IRAF}^{4}$ procedures for long-slit spectroscopy. All images were biassubtracted and then flat-field corrected using dome flats. The background was interpolated by fitting the region on both sides of the spectrum with a low-order polynomial and then subtracted. Extractions were usually weighted by the variance based on the data values and a Poisson/CCD model using the gain and readout noise parameters 5 .

Spectra were wavelength-calibrated by means of reference arc spectra, and the resulting wavelength-calibrated spectra were checked by measuring the position of known bright-night skylines (usually the [OI] lines at $\lambda 5577 \AA$ and $\lambda 6300 \AA$ ). Small discrepancies have been corrected by applying a rigid shift in order to match the skylines wavelengths. The spectra were fluxcalibrated using a spectroscopic standard star observed with the same telescope setup and reduced as was the supernova itself. If spectrophotometric standard stars were not available for a given epoch, response curves obtained for the same telescope/instrument in another night close in time were used. The flux calibration was then checked against the photometry and, when necessary, spectral fluxes were scaled to match the photometric data. Partial correction for atmospheric absorption was also applied. Multiple spectra of the SN obtained in the same night were combined in order to improve the signal-to-noise ratio and almost simultaneous spectra covering different but overlapping spectral ranges were merged together in a single, more extended spectrum. When the observing conditions were not photometric, spectra covering different wavelength ranges were scaled to match in the overlapping region.

There was good pre-maximum spectrophotometric coverage, which is one of the main goals of the European Supernova Collaboration. In fact, soon after the explosion, Type Ia SNe show differences that are bigger than those at later phases, hence early observations can provide useful hints for understanding the nature of the progenitors and the explosion mechanism. The spectral evolution of SN 2004dt from day -10 to day +1 is shown in Fig. 2, while the postmaximum spectral evolution is shown in Fig. 3.

The good temporal coverage allows us to follow the fast evolution at early times on a daily basis. Early spectra exhibit an unusually broad and asymmetric Si II $\lambda 6355 \AA$ feature, analogous with SN 2005cf (Garavini et al. 2007), which gradually shifts to the red and becomes narrower at later phases. Strong absorption features identified as Ca II, S II, Mg II, and O I are also evident. A more careful analysis shows that almost all absorption troughs, except for the S II $\lambda \lambda 5454,5640 \AA$, and Si III $\lambda 4565 \AA$, are highly blueshifted, as discussed in details in Sect. 4. A possible line identification coming from the spectral modeling is shown in Sect. 5.1.

\section{Light-curve parameters}

The photometric evolution of SN2004dt will be discussed in detail in a forthcoming paper, while here we report some preliminary estimates of the relevant photometric parameters. A

\footnotetext{
4 http://iraf.noao.edu/

IRAF is distributed by the National Optical Astronomy Observatories (NOAO), which are operated by the Association of Universities for Research in Astronomy (AURA), Inc., under cooperative agreement with the National Science Foundation.

5 Variance weighting is often called "optimal" extraction since it produces the best unbiased signal-to-noise estimate of the flux in the 2D profile (Horne 1986; Marsh 1989).
} 
Table 2. Log of the optical spectroscopic observations of SN 2004dt.

\begin{tabular}{|c|c|c|c|c|c|c|}
\hline$\overline{\text { Date }}$ & $\begin{array}{c}\text { JD } \\
-2400000 \\
\end{array}$ & $\begin{array}{r}\text { Epoch }^{\star} \\
\text { [days] }\end{array}$ & $\begin{array}{c}\text { Approx. range } \\
[\AA]] \\
\end{array}$ & $\begin{array}{l}\text { Telescope } \\
\text { +Instrument }\end{array}$ & $\begin{array}{l}\text { Flux } \\
\text { standard }\end{array}$ & Reference \\
\hline$\overline{2004 / 08 / 12}$ & 53230.22 & -10.1 & $4500-10300$ & ANU2.3+DBS & hr718 & M. Salvo \\
\hline 2004/08/12 & 53230.67 & -9.6 & $3200-8600$ & CA2.2+CAFOS & $b d+284211$ & G. Pignata \\
\hline 2004/08/13 & 53231.23 & -9.1 & 4000-9700 & ANU2.3+DBS & hr718 & M. Salvo \\
\hline 2004/08/13 & 53231.63 & -8.7 & $3250-9150$ & CA2.2+CAFOS & bd+284211 & G. Pignata \\
\hline 2004/08/15 & 53233.64 & -6.7 & $3200-10000$ & CA2.2+CAFOS & bd+284211 & G. Pignata \\
\hline $2004 / 08 / 15$ & 53233.69 & -6.6 & $3600-8900$ & NOT+ALFOSC & Feige110 & V. Stanishev \\
\hline $2004 / 08 / 16$ & 53233.71 & -6.6 & $3100-10200$ & TNG+DOLORES & - & N. de la Rosa \\
\hline $2004 / 08 / 16$ & 53234.57 & -5.7 & $3200-9600$ & CA2.2+CAFOS & $b d+284211$ & G. Pignata \\
\hline 2004/08/17 & 235.69 & -4.6 & $3300-9700$ & NOT+ALFOSC & $b d+284211$ & V. Stanishev \\
\hline 2004/08/18 & 236.54 & -3.8 & $3200-9800$ & CA2.2+CAFOS & bd+284211 & G. Pignata \\
\hline 2004/08/19 & 3237.18 & -3.1 & $3300-9000$ & ANU2.3+DBS & Feige110 & M. Salvo \\
\hline $2004 / 08 / 20$ & 53238.22 & -2.1 & $3300-8800$ & ANU2.3+DBS & Feige110 & M. Salvo \\
\hline $2004 / 08 / 21$ & 53239.23 & -1.1 & $3300-8800$ & ANU2.3+DBS & Feige110 & M. Salvo \\
\hline $2004 / 08 / 21$ & 53239.59 & -0.7 & $3400-7700$ & ASIAGO+AFOSC & $b d+254655$ & S. Benetti \\
\hline $2004 / 08 / 23$ & 53241.28 & +1.0 & $3500-7200$ & ANU2.3+DBS & Feige110 & M. Salvo \\
\hline $2004 / 08 / 24$ & 3242.28 & 2.0 & $3500-7200$ & ANU2.3+DBS & Feige 110 & M. Salvo \\
\hline $2004 / 08 / 24$ & 3242.59 & 2.3 & $3300-9000$ & NOT+ALFOSC & bd+284211 & V. Stanishev \\
\hline $2004 / 08 / 25$ & 53243.68 & 3.4 & 3300-9100 & CA2.2+CAFOS & bd+284211 & G. Pignata \\
\hline 2004/08/26 & 53244.15 & 3.8 & $3250-9150$ & CA2.2+CAFOS & bd+284211 & G. Pignata \\
\hline 2004/08/31 & 53249.58 & 9.3 & $3300-7900$ & TNG+DOLORES & HR9087 & N. de la Rosa \\
\hline 2004/09/05 & 53254.58 & 14.3 & $3350-9000$ & NOT+ALFOSC & - & V. Stanishev \\
\hline 2004/09/08 & 53257.63 & 17.3 & $3400-7700$ & ASIAGO+AFOSC & $b d+254655$ & N. de la Rosa \\
\hline 2004/09/12 & 53260.69 & 20.4 & $3500-8900$ & NOT+ALFOSC & - & V. Stanishev \\
\hline 2004/09/18 & 53267.19 & 26.9 & $3500-9000$ & ANU2.3+DBS & Feige 110 & J. Rich \\
\hline 2004/09/19 & 53268.15 & 27.9 & $3500-9000$ & ANU2.3+DBS & Feige 110 & J. Rich \\
\hline 2004/09/28 & 53277.58 & 37.3 & $3400-9000$ & NOT+ALFOSC & - & V. Stanishev \\
\hline 2004/10/08 & 53287.48 & 47.2 & $3500-9200$ & CA2.2+CAFOS & Feige 110 & G. Pignata \\
\hline $2004 / 10 / 22$ & 53301.09 & 60.8 & $3400-9000$ & ANU2.3+DBS & Feige 110 & M. Salvo \\
\hline $2005 / 01 / 21-22$ & 53392.80 & 152.5 & $3500-8600$ & CA2.2+CAFOS & $\begin{array}{l}\text { Feige } 34 \\
\text { G191-B2B }\end{array}$ & $\begin{array}{r}\text { A. Pastorello } \\
\text { S. Taubenberger }\end{array}$ \\
\hline 2005/08/11 & 53593.78 & 353.5 & $4000-8400$ & VLT+FORS1 & GD50 & $\begin{array}{r}\text { N. de la Rosa } \\
\text { S. Benetti }\end{array}$ \\
\hline
\end{tabular}

* Relative to the $B$ band maximum. ${ }^{*}$ ANU: Australian National University 2.3-m telescope, DBS: Double Beam Spectrograph; CA2.2: Calar Alto 2.2-m telescope, CAFOS: Calar Alto Faint Object Spectrograph; NOT: Nordic Optical Telescope (2.5-m), ALFOSC Andalucia Faint Object Spectrograph and Camera; TNG: Telescopio Nazionale Galileo (3.6-m), DOLORES: Device Optimized for the LOw RESolution, ASIAGO: 1.8-m Copernico telescope AFOSC: Asiago Faint Object Spectrograph and Camera, VLT: ESO Very Large Telecope, Unit Telescope 2 (8.2 m), FORS1: FOcal Reducer/low dispersion Spectrograph.

preliminary analysis of the optical light curves gives $B_{\max }=$ $15.33 \pm 0.02$ on Aug. 22nd (JD = $2453240.3 \pm 0.5)$. The decline rate after maximum is $\Delta m_{15}(B)=1.21 \pm 0.05$. After the correction for the Galactic extinction $\left(A_{B}=0.109 \mathrm{mag}\right.$, as measured by Schlegel et al. 1998), we obtain $(B-V)_{\max }=-0.03 \pm 0.02$, corresponding to $E(B-V)_{\max }=0.04 \pm 0.04$, which is consistent with negligible reddening. This is derived from the relation $(B-V)_{\max }=0.09( \pm 0.08) \times\left(\Delta m_{15}-1.1\right)-0.08( \pm 0.03)$, (Altavilla et al. 2004), which gives $(B-V)_{\max }=-0.07 \pm 0.04$. According to the Lira relation (Lira 1995), we find $E(B-V)_{\text {tail }}=0.11 \pm 0.03$. The weighted average color excess is $E(B-V)_{\text {avg }}=0.08 \pm 0.05$. The discrepancies between the two values of the color excess can be due to the SN spectroscopic peculiarities that affect the color evolution and make the usual reddening law fail or lose accuracy. The absence of clearly detectable Na I D lines in the spectra supports the hypothesis of a small reddening, if any. In this case the absolute $B$ magnitude at maximum would be $B_{\max } \sim-19.4$ $\left(H_{0}=70 \mathrm{~km} \mathrm{~s}^{-1} \mathrm{Mpc}^{-1}\right)$.

\section{Expansion velocities and spectral properties derived from early spectra}

By looking at the early spectra, the peculiar large, asymmetric and flat-bottomed Si II $\lambda 6355 \AA$ feature is immediately evident.
The comparison of the SN 2004dt spectrum 10 days before maximum with SN 1994D (Patat et al. 1996) at the same epoch shows the peculiarity of the SN 2004dt spectrum and a high blue shift of the Si II $\lambda 6355 \AA$ becomes evident, too (Fig. 4). The peculiarities of SN 2004dt also persist later on, as shown in Fig. 5, where spectra of SN 2004dt at different epochs are compared with those of the normal SN Ia 1996X (Salvo et al. 2001) and with other two HVG SNe: SN 2002bo (Benetti et al. 2004) and SN 2002er (Pignata et al. 2004; Kotak et al. 2005).

The analysis of the very early (epoch -10) spectrum gives the following results. A strong absorption, measured at $\lambda 3930 \AA$, was initially identified as C II $\lambda 4267 \AA$ (Mazzali 2001; Branch et al. 2003; Thomas et al. 2007), implying an expansion velocity of about $23700 \mathrm{~km} \mathrm{~s}^{-1}$. In this hypothesis the other intense $\mathrm{C}_{\text {II }}$ $\lambda \lambda 6576-6583 \AA$ transitions are blended with the Si II $\lambda 6355 \AA$, which indeed appears very broad and intense and has an asymmetric absorption trough. On the other hand, there was no strong evidence of the C II $\lambda \lambda 7231,7236 \AA$ line (this line, however, is expected to be much weaker, Mazzali 2001). A second hypothesis was that the $\lambda 3930 \AA$ feature was a strong absorption typically attributed to Si II $\lambda \lambda 4128,4131 \AA$ (Pskovskii 1969), implying an expansion velocity of about $14500 \mathrm{~km} \mathrm{~s}^{-1}$. Synthetic spectra (see Sect. 5) can reproduce the observations without 


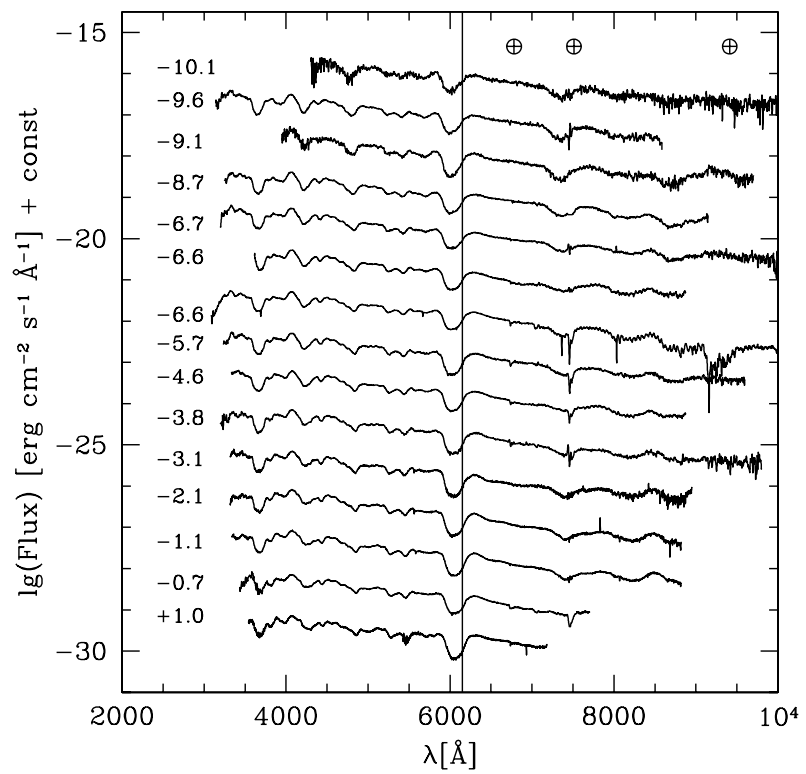

Fig. 2. Early spectral sequence for SN 2004dt. The vertical line at $\lambda=$ $6150 \AA$ makes the evolution of the blueshifted Si II $\lambda 6355 \AA$ feature clear. Spectra are restframe. No reddening correction has been applied. The $\oplus$ symbols mark the position of the main telluric absorptions, which have been partially removed. Spectra have been shifted downwards for clarity (shift step $=-1$ ). The phase with respect to the maximum of the B light curve is shown to the left of each spectrum.

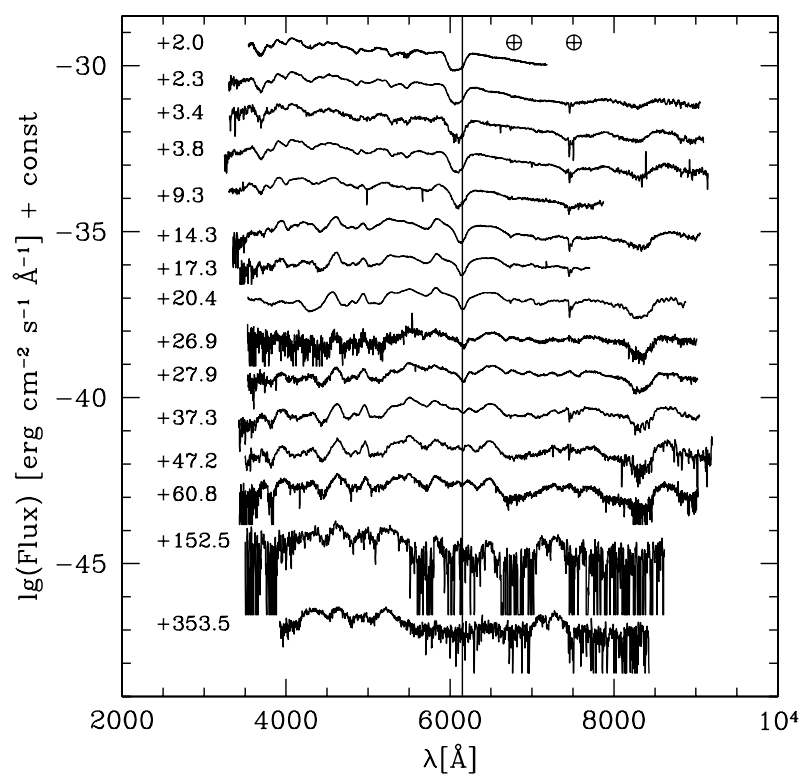

Fig. 3. SN 2004dt post maximum spectral evolution. Labels as in Fig. 2.

using $\mathrm{C}$, thus rejecting the first hypothesis and supporting the Si line identification.

The Si II $\lambda 6355 \AA$ minimum at $6000 \AA$ gives an expansion velocity of about $16700 \mathrm{~km} \mathrm{~s}^{-1}$ and the O I $\lambda 7773 \AA$ line (whose minimum is at $7340 \AA$ ) also gives a similar expansion velocity of $16700 \mathrm{~km} \mathrm{~s}^{-1}$. The Si II $\lambda 6355 \AA$ expansion velocity measured for SN 1994D at similar epochs (Patat et al. 1996) is significantly lower: $\sim 14000 \mathrm{~km} \mathrm{~s}^{-1}$ at -11 days and $\sim 13000 \mathrm{~km} \mathrm{~s}^{-1}$ at -10 days. The $\mathrm{Ca}$ II $\mathrm{H} \& \mathrm{~K}$ line is characterized by a velocity of about $22500 \mathrm{~km} \mathrm{~s}^{-1}$, equal to the Ca II H\&K velocity measured for SN 1994D at day -10. In Fig. 6, the Si II $\lambda 6355 \AA$ velocity evolution is shown and compared with those of other

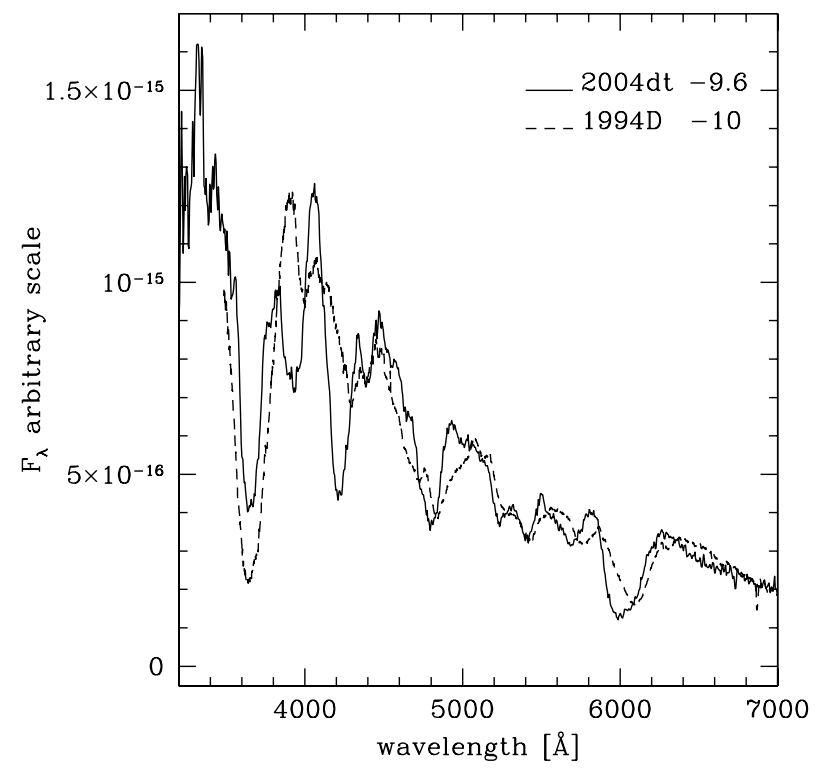

Fig. 4. Comparison among spectra of SN 2004dt (solid line) and SN 1994D (dashed line) $\sim 10$ days before maximum. To be noticed the very different blue-shift of the Si II $\lambda 6355 \AA$ features and the more similar blue-shift of the S II $\lambda 5640 \AA$ A lines. Significant differences are present in the 3800-4200 ̊ region.

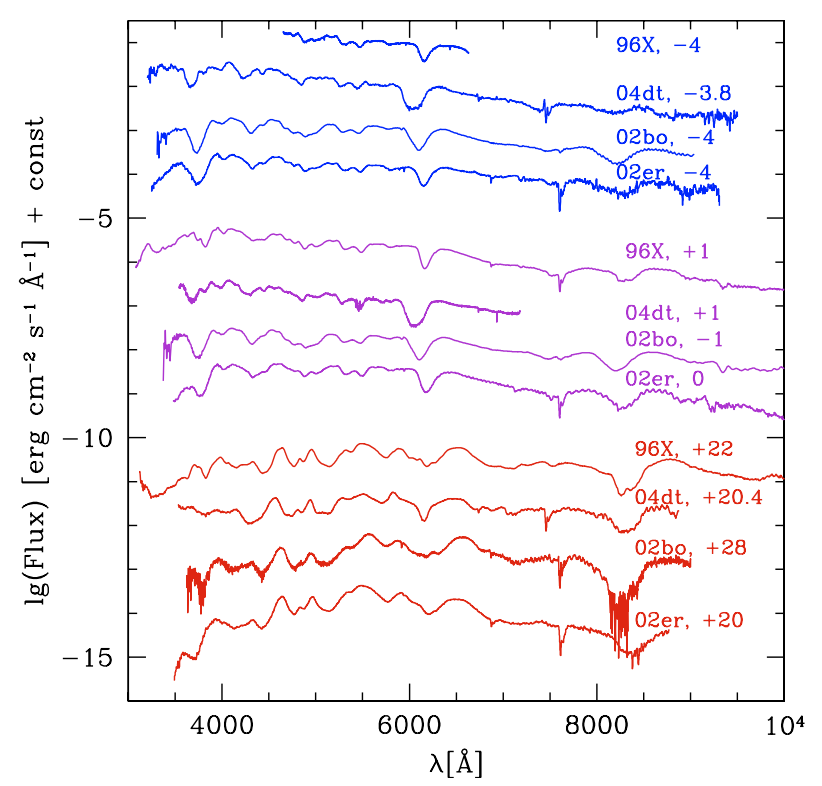

Fig. 5. Comparison among spectra of SN 2004dt, SN 1996X, SN 2002bo, and SN2002er at three different epochs (premaximum, maximum, postmaximum).

SNe Ia. The fast decline in the Si II velocity is evident, and corresponds to a decline rate of $\dot{v}=160 \pm 15 \mathrm{~km} \mathrm{~s}^{-1} \mathrm{~d}^{-1}$, where $\dot{v}=-\Delta v / \Delta t$ is the average daily rate of decrease in the expansion velocity after maximum (Benetti et al. 2005). Lentz et al. (2000) computed the Si II $\lambda 6355 \AA$ expected velocity evolution in $\mathrm{SNe}$ Ia by varying the metallicity in the $\mathrm{C}+\mathrm{O}$ layer in the standard deflagration model W7 (Nomoto et al. 1984; Thielemann et al. 1986). The expected trends for 3 different metallicities are also shown in Fig. 6. The high expansion velocity of SN 2004dt, as well as those of SN 2002 bo or SN 1984A, cannot be reproduced even with unreasonable high metallicity values, since the unmixed W7 model predicts no $\mathrm{Si}$ at velocities higher than 


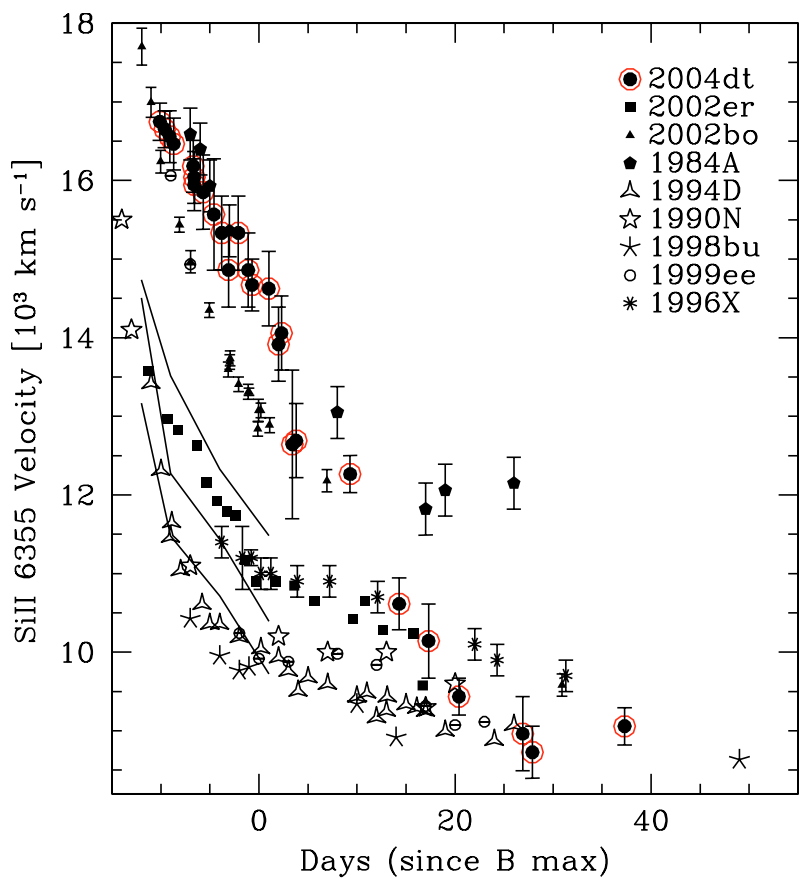

Fig. 6. The Si II $\lambda 6355 \AA$ A expansion velocity evolution as deduced from the position of the absorption minimum, compared with other $\mathrm{SNe}$ and with the expected evolution for different metallicities (solid line, top: $\times 10$ solar metallicity; middle: $\times 1$; bottom: $\times 0.1$ ) obtained by Lentz et al. (2000).

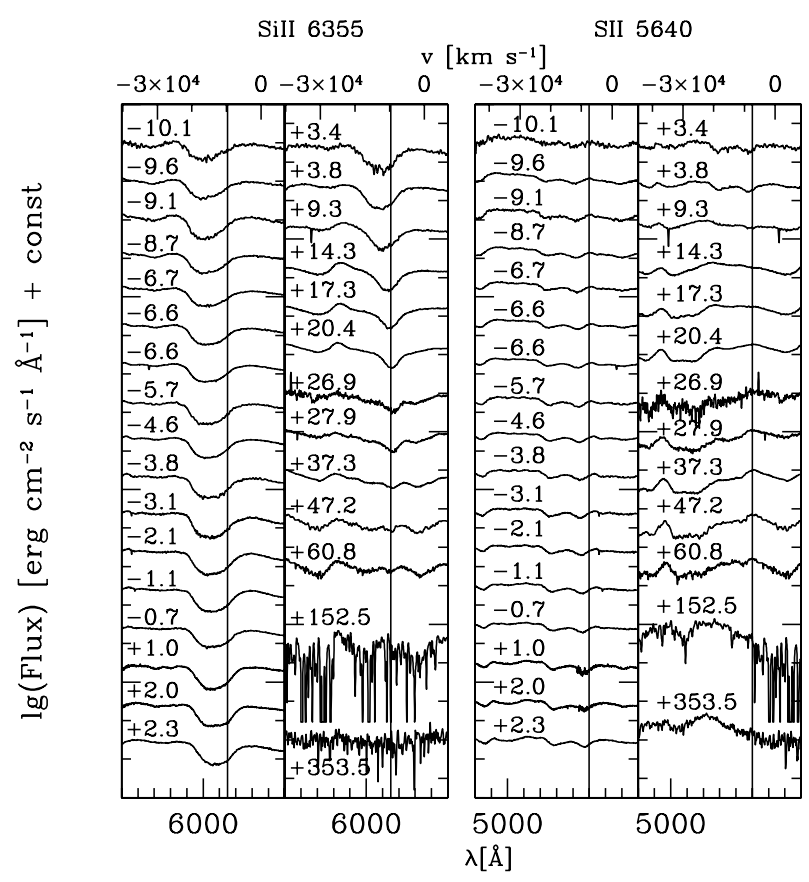

Fig. 7. Evolution with time of the Si II $\lambda 6355 \AA$ (left panel) and S II $\lambda \lambda 5454,5640 \AA$ (right panel). Vertical lines at 6150 and $5500 \AA$ are shown to guide the eye.

$\sim 15000 \mathrm{~km} \mathrm{~s}^{-1}$. The evolution in time of the Si II $\lambda 6355 \AA$ line shape is shown in Fig. 7. The line shows a wide flat-bottomed profile until some days past maximum, the first spectrum with a narrower profile being the one taken at about 2 weeks past maximum.

In Fig. 8 the CaII H\&K velocity evolution is shown and compared with those of other SNe Ia. However, the Ca II H\&K

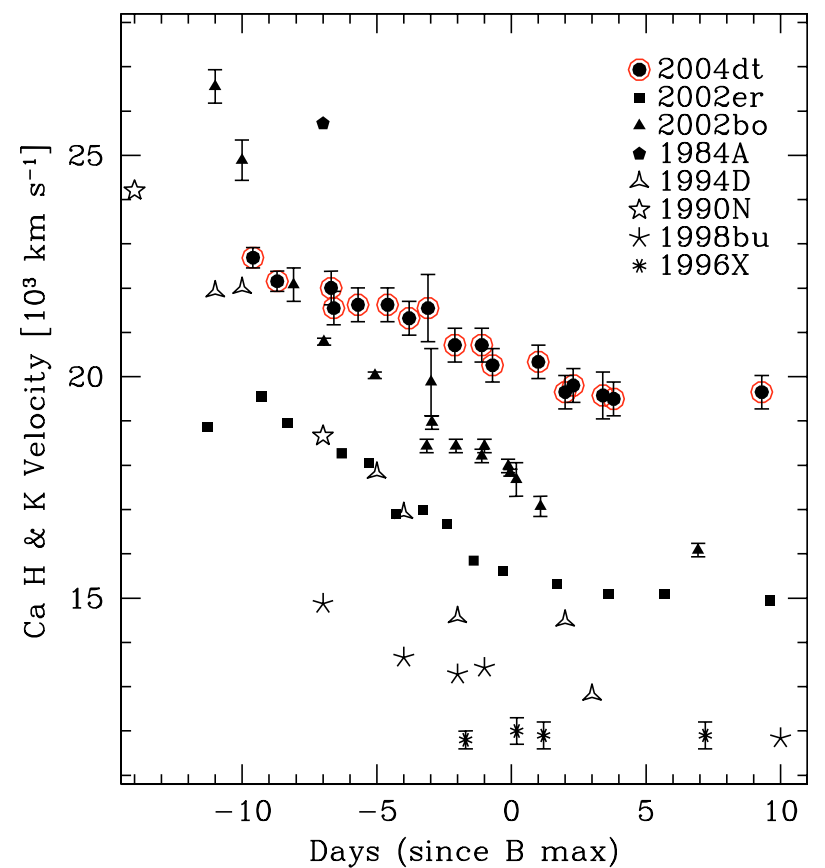

Fig. 8. The Ca II $\mathrm{H} \& \mathrm{~K}$ expansion velocity as deduced from the position of its minimum, compared with those of other SNe.

feature could be dominated by the blended Si II $\lambda \lambda 3856,3863 \AA$ line, while the Si II $\lambda 4130 \AA$ is well-separated. In this case, the corresponding Si II $\lambda \lambda 3856,3863 \AA$ velocity is significantly lower $\left(\leq 16150 \mathrm{~km} \mathrm{~s}^{-1}\right)$ than the one computed for Ca II H\&K, and more like to the Si II $\lambda 6355 \AA$ values, thus supporting this hypothesis. Moreover, the comparison of the spectra with a theoretical model (discussed in Sect. 5) reinforces this second hypothesis. Ca II H\&K contamination by Si II has already been suggested by Kirshner et al. (1993), who pointed out that the Si II $\lambda 3858$ is fainter, but comparable, to the Ca II H\&K feature, and by Nugent et al. (1997), who suggested that the "split" just blueward of the $\mathrm{Ca}$ II $\mathrm{H} \& \mathrm{~K}$ feature visible in both the observed spectrum at maximum of SN 1994D and the corresponding W7 synthetic spectra, could likely be explained by a blend with Si II 13858. Lentz et al. (2000) confirmed the identification of the blue wing with the Si II line. Several other SNe, as for example SN 1990N, SN 1996X, and probably SN 1998bu, and SN 2003du show this contamination, too (Stanishev et al. 2006).

SN 2004dt expansion velocities for Si II $\lambda 6355 \AA$, Si II $\lambda \lambda 3856,3863 \AA$, and Ca II H\&K are tabulated in Table 3 . The large uncertainties are mainly due to the wide and flat-bottomed profile of the lines. The Si II $\lambda \lambda 5958,5979 \AA$ feature gives an expansion velocity of $\sim 14300 \mathrm{~km} \mathrm{~s}^{-1}$, and if we assume the line at $4395 \AA$ to be Si III $\lambda 4565 \AA$, we obtain a lower velocity: $11200 \mathrm{~km} \mathrm{~s}^{-1}$ (to be compared with the $\sim 17000 \mathrm{~km} \mathrm{~s}^{-1}$ of the Si II $\lambda 6355 \AA$ ). If the Si III identification is correct, it could be proof of high ionization temperature in the ejecta. The Mg II $\lambda 4481 \AA$ minimum gives an expansion velocity of $17900 \mathrm{~km} \mathrm{~s}^{-1}$, similar to the other HVFs. The S II $\lambda \lambda 5454,5640 \AA$ (the typical "W" feature) gives a velocity of about $12000 \mathrm{~km} \mathrm{~s}^{-1}$, significantly lower than what is measured for the Si II lines. The evolution of this feature with time is shown in Fig. 7; it can be followed until $\sim 4$ days after maximum and has disappeared in the next available spectrum taken about 2 weeks after maximum. The lower velocities measured for Si III $\lambda 4565 \AA$ and S II $\lambda \lambda 5454,5640 \AA$ suggest that these ions are mainly present in a 
Table 3. Expansion velocities from the Si II $6355 \AA$ A, Ca II H\&K, or Si II $3860 \AA$ A and S II $5640 \AA$ A absorption.

\begin{tabular}{|c|c|c|c|c|}
\hline $\begin{array}{l}\text { Phase } \\
\text { [day] }\end{array}$ & $\begin{array}{r}\text { Si II } 6355 \\
{\left[\mathrm{~km} \mathrm{~s}^{-1}\right]}\end{array}$ & $\begin{array}{c}\mathrm{H} \& \mathrm{~K}^{\bullet} \\
{\left[\mathrm{km} \mathrm{s}^{-1}\right]}\end{array}$ & $\begin{array}{r}\text { Si II } 3860^{\circ} \\
{\left[\mathrm{km} \mathrm{s}^{-1}\right]}\end{array}$ & $\begin{array}{c}\text { S II } 5640 \\
{\left[\mathrm{~km} \mathrm{~s}^{-1}\right]}\end{array}$ \\
\hline-10.1 & $16750(235)$ & - & & $12230(531)$ \\
\hline-9.6 & $16650(235)$ & 22690 (227) & $16150(233)$ & $12390(266)$ \\
\hline-9.1 & $16560(330)$ & - & $15610(233)$ & $11750(372)$ \\
\hline-8.7 & $16460(330)$ & 22160 (227) & - & 11800 (266) \\
\hline-6.7 & $16180(330)$ & 22000 (379) & $15460(388)$ & $11380(213)$ \\
\hline-6.6 & $15940(330)$ & 21550 (379) & $14990(388)$ & $11270(213)$ \\
\hline-6.6 & $16040(330)$ & - & - & 11270 (266) \\
\hline-5.7 & $15850(471)$ & 21630 (379) & 15070 (388) & 11060 (372) \\
\hline-4.6 & 15570 (707) & 21630 (379) & $15070(388)$ & $10740(160)$ \\
\hline-3.8 & $15330(471)$ & 21320 (379) & $14760(388)$ & 10470 (266) \\
\hline-3.1 & $14860(471)$ & 21550 (758) & 14990 (777) & $10370(213)$ \\
\hline-2.1 & $15330(471)$ & 20710 (379) & $14140(388)$ & 9993 (266) \\
\hline-1.1 & $14860(471)$ & 20710 (379) & $14140(388)$ & 9834 (266) \\
\hline-0.7 & 14670 (330) & 20260 (379) & 13670 (388) & 9568 (266) \\
\hline+1.0 & $14620(471)$ & 20340 (379) & 13750 (388) & $9036(532)$ \\
\hline+2.0 & $13920(471)$ & 19650 (379) & 13050 (388) & 9036 (266) \\
\hline+2.3 & $14060(471)$ & 19800 (379) & 13200 (388) & 9036 (213) \\
\hline+3.4 & $12640(943)$ & $19580(531)$ & $12970(543)$ & 8505 (372) \\
\hline+3.8 & $12690(471)$ & 19500 (379) & $12890(388)$ & 8877 (266) \\
\hline+9.3 & $12270(235)$ & 19650 (379) & 13050 (388) & $8133(532)$ \\
\hline+14.3 & $10610(330)$ & - & - & - \\
\hline+17.3 & $10140(471)$ & - & - & - \\
\hline+20.4 & 9435 (235) & - & - & - \\
\hline+26.9 & 8963 (471) & - & - & - \\
\hline+27.9 & 8727 (330) & - & - & - \\
\hline+37.3 & 9057 (235) & - & - & - \\
\hline
\end{tabular}

- See discussion is Sect. 4.

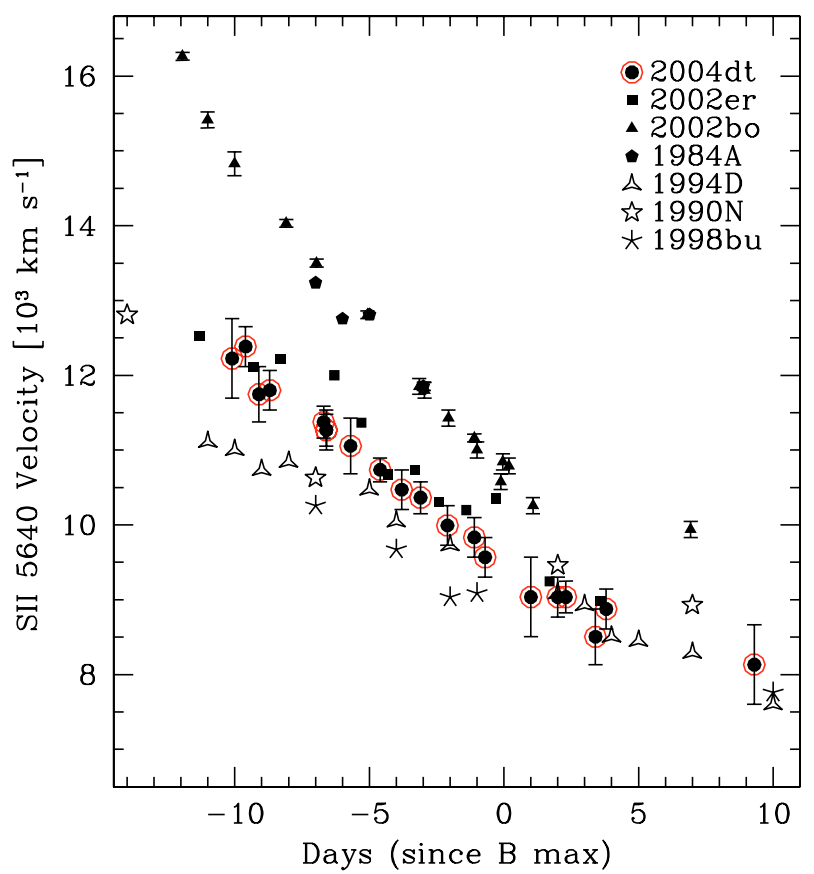

Fig. 9. The S II $\lambda 5640 \AA$ expansion velocity as deduced from its minimum, compared with those of other $\mathrm{SNe}$.

lower-velocity region with respect to Si II, Ca II, O I, and $\mathrm{Mg}$ II. The S II $\lambda 5454,5640 \AA$ velocity evolution is shown in Fig. 9 . Interestingly enough in this plot, SN 2004dt is not one of the extreme objects as in the previous plots (Figs. 6, 8), and it is quite similar to SN 2002er, and SN 1994D (see also Fig. 4).

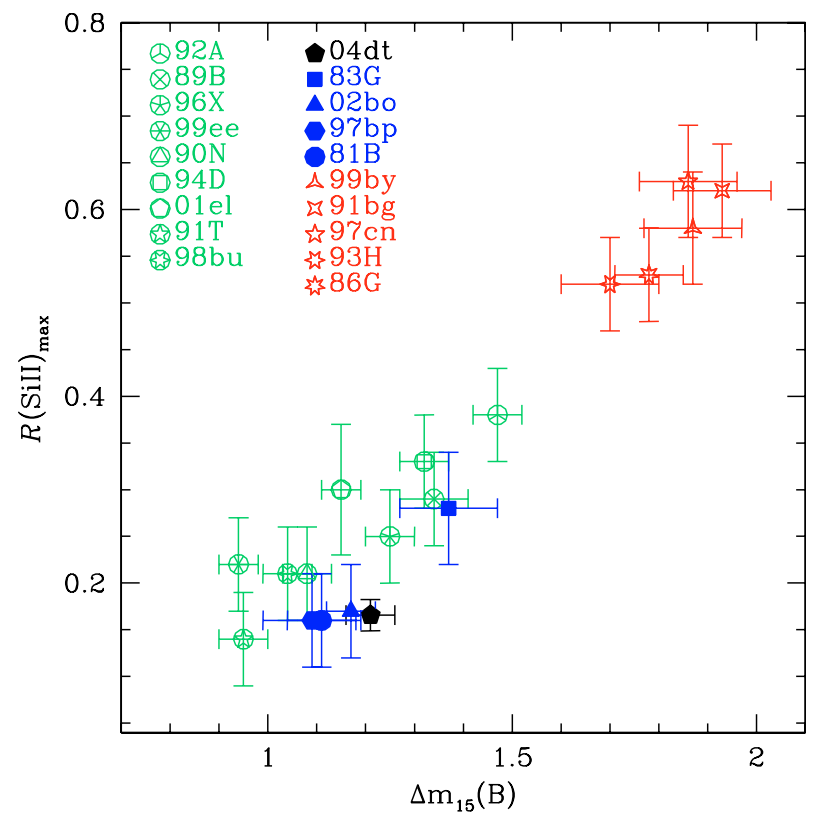

Fig. 10. $\mathcal{R}$ (Si II) vs. $\Delta m_{15} B$ for $\mathrm{SN} 2004 \mathrm{dt}$ and a sample of $\mathrm{SNe}$ shown for comparison. Filled symbols refer to HVG SNe, starred symbols to FAINT SNe, and all the others to LVG SNe, as defined in Benetti et al. (2005).

The Ca II IR triplet gives a velocity of about $16500 \mathrm{~km} \mathrm{~s}^{-1}$, but there is a hint of a bluer component whose minimum at $\sim 8000 \AA$ corresponds to an expansion velocity of $21000 \mathrm{~km} \mathrm{~s}^{-1}$. These values can be compared to the velocity measured for the two components and at similar phases for SN 2002dj (17 700-27600 $\mathrm{km} \mathrm{s}^{-1}$ at -11 days), SN 2001el (17 100$23800 \mathrm{~km} \mathrm{~s}^{-1}$ at -9 days), SN 2003du (15 500-22500 $\mathrm{km} \mathrm{s}^{-1}$ at -11 days), SN 2003kf (12600-23500 $\mathrm{km} \mathrm{s}^{-1}$ at -9 days), SN 2002er (15600-23100 $\mathrm{km} \mathrm{s}^{-1}$ at -7 days), SN 2002bo (14900-22 $100 \mathrm{~km} \mathrm{~s}^{-1}$ at -8 days), and SN 2003cg (12 700$22000 \mathrm{~km} \mathrm{~s}^{-1}$ at -8.5 days) (data from Mazzali et al. 2005b). Like SN 2004dt, all these objects show HVFs in the Ca II IR triplet. HVFs are best detected in very early spectra (earlier than $\sim 1$ week before $B$ maximum) in the Ca II IR triplet but also in the Si II $\lambda 6355 \AA$ line. They are supposed to arise from abundance or density enhancements (see Sect. 5).

As shown by Nugent et al. (1995), there is a correlation between the ratio $\mathcal{R}(\mathrm{Si}$ II) of the depth of the Si II $\lambda 5972 \AA$ and Si II $\lambda 6355 \AA$ absorption troughs near maximum with the decline speed of the $B$ light curve (i.e. the luminosity), in the sense that higher values of $\mathcal{R}(\mathrm{Si}$ II) correspond to subluminous, fastdeclining events (but the correlation fails for $\Delta m_{15}(B) \lesssim 1.2$, Benetti et al. 2004). We measured $\mathcal{R}(\mathrm{Si}$ II $) \sim 0.16$ (day +1$)$. This value, together with the estimate of $\Delta m_{15}(B)=1.21$ falls within the HVG supernova area in the $\mathcal{R}(\mathrm{Si}$ II $)-\Delta m_{15}(B)$ plot (Fig. 10 , see also Benetti et al. 2005). This result is also confirmed by the $\dot{v}-\Delta m_{15}(B)$ plot (Fig. 11, see also Benetti et al. 2005) in which the HVG, low-velocity gradient (LVG) and faint SNe are separated better than in Fig. 10.

This plot shows that SN $2004 \mathrm{dt}$ has the highest $\dot{v}$ in our sample $(\dot{v}=160 \pm 15)^{6}$. Also, the evolution of $\mathcal{R}(\mathrm{Si}$ II) (Fig. 12) is

6 The most similar obiect to SN 2004dt is SN 1983G $(\dot{v}=125 \pm 20$, Benetti et al. 2005). More recent measurements show that HVG SNe may have $\dot{v}$ well above 100, and in particular SN 1983G is characterized by $\dot{v} \sim 148$ (Benetti, private communication), slighlty higher than the value reported in the plot, and closer to SN 2004dt value. 


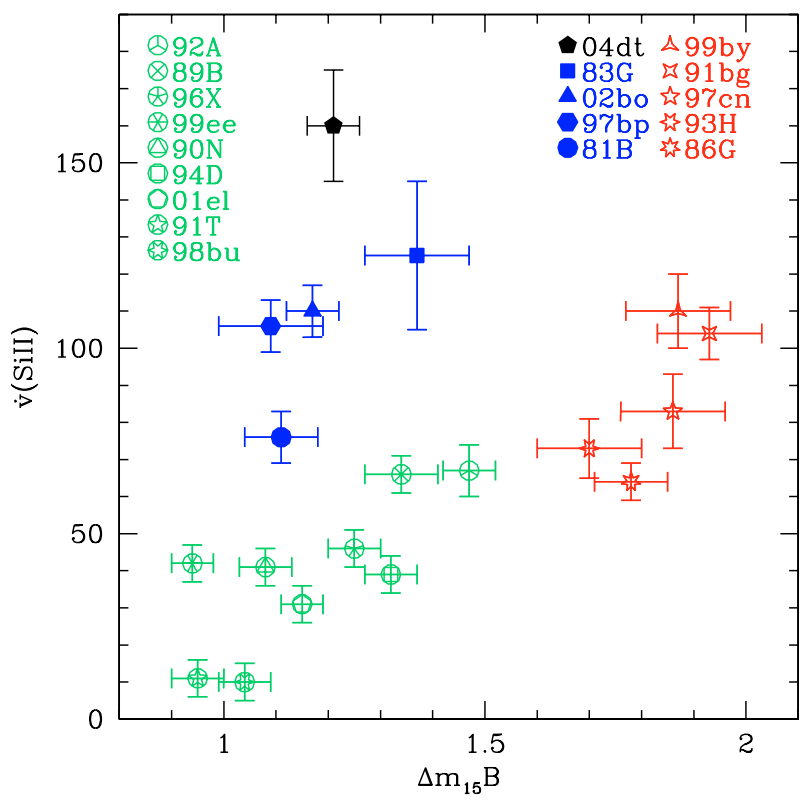

Fig. 11. $\dot{v}$ vs. $\Delta m_{15} B$ for SN 2004dt and a sample of SNe shown for comparison. Filled symbols refer to HVGSNe, starred symbols to FAINT $\mathrm{SNe}$, and all the others to LVGSNe, as defined in Benetti et al. (2005).

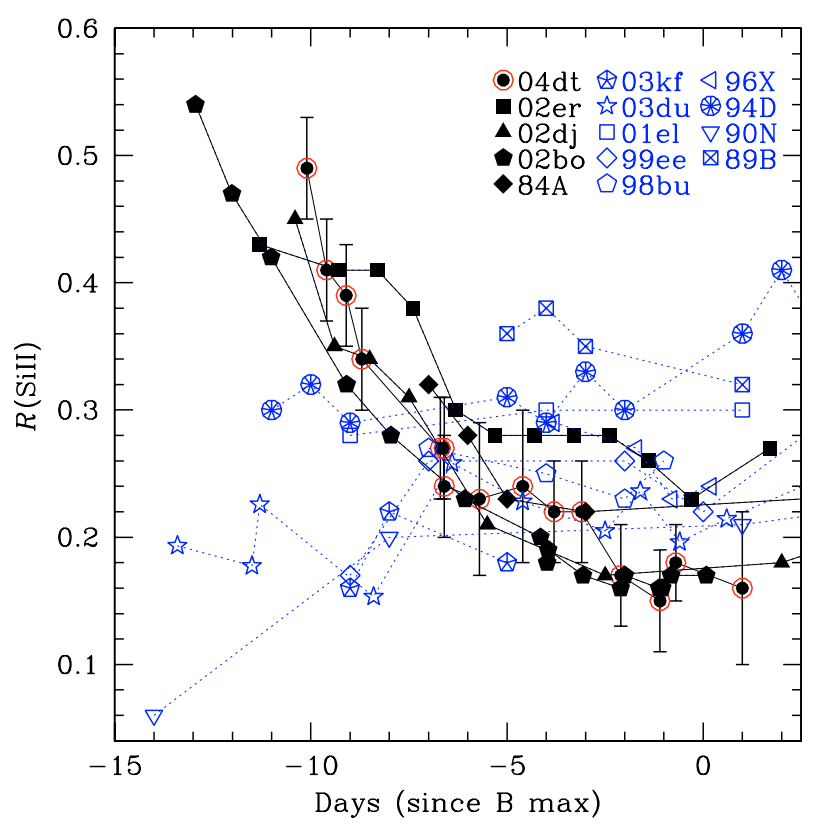

Fig. 12. Premaximum temporal evolution of the $\mathcal{R}(\mathrm{Si}$ II) parameter for SN 2004dt and a sample of SNe shown for comparison. Filled symbols refer to HVG SNe, open symbols to LVGSNe, as defined in Benetti et al. (2005).

very similar to the HVG SNe as shown in Benetti et al. (2005). In conclusion, SN 2004dt seems to belong to the "high-velocity" SNe Ia, such as SN 2002bo (Benetti et al. 2004), SN 2002dj (Pignata et al. 2005) or SN 2002er (Kotak et al. 2005; Pignata et al. 2004). Nevertheless, SN 2004dt also shows some peculiarities with respect to the HVGSNe (Fig. 5, Fig. 7 left panel), in particular the Si II ( 26355$)$ feature remains broader and probably visible for a longer time than for most of HVG SNe $(>28 \mathrm{~d})$. However, even if they are kinematically different from normal SNe Ia, their photometric properties, such as the peak luminosity and the light-curve decline $\left(\Delta m_{15}\right)$, do not show any peculiarity,

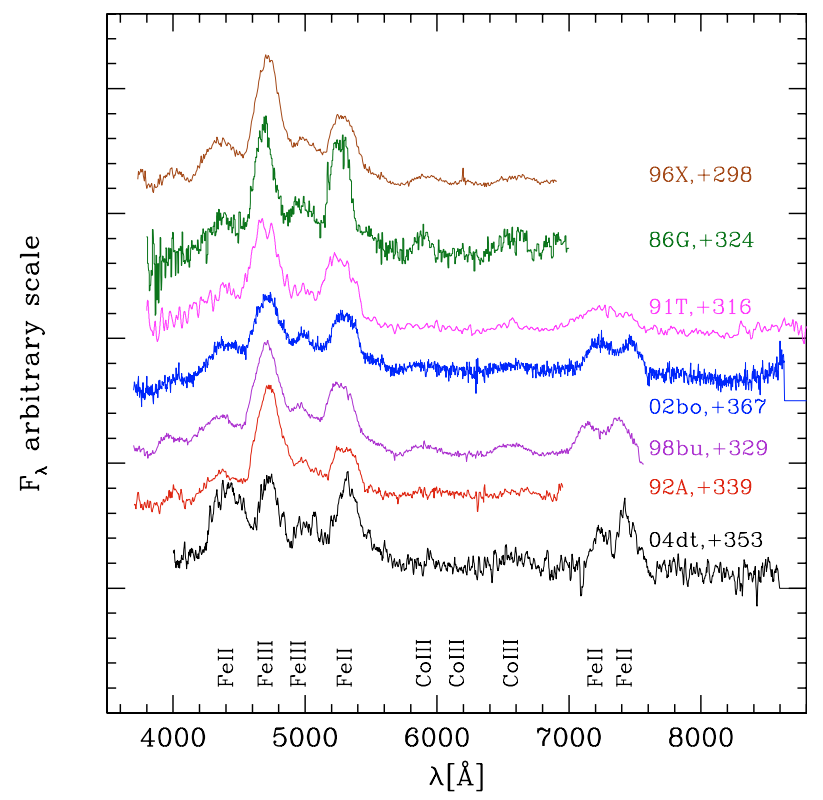

Fig. 13. Comparison of late spectra ( 1 year past maximum) of different SNe, including the peculiar SN 1991T and SN 1986G. At this phase, SN 1991T and SN 1998 bu have not yet been affected by the light echo contamination (Schmidt et al. 1994; Cappellaro et al. 2001). Spectra have been normalized and shifted for clarity. The phase with respect to the maximum of the $B$ light curve is shown close to the name. SN 2004dt spectrum has been slightly smoothed.

although the color evolution can be affected, as mentioned in Sect. 3 (see also Benetti et al. 2004).

Even if the present work is focused on the early spectral evolution, it is worth short comment here on the very last spectrum in our sample, taken almost one year after maximum (day +353). At this phase, when the ejecta have become thin and transparent to radiation, spectra are usually dominated by the strong emission lines of $\mathrm{Fe}[\mathrm{II}]$ and $\mathrm{Fe}$ [III] originating from the radioactive decay of ${ }^{56} \mathrm{Ni}$ into stable ${ }^{56} \mathrm{Fe}$ through ${ }^{56} \mathrm{Co}$ (Kuchner 1994).

Figure 13 shows the comparison of a late spectrum of SN 2004dt with spectra of other $\mathrm{SNe}$ at a similar phase (SN 1996X, Salvo et al. 2001; SN 1986G, Cristiani et al. 1992; SN 1991T, Gómez \& López 1998; SN 1998bu, Cappellaro et al. 2001; SN 1992A, ESO+Asiago SN archive; SN 2002bo, ESO+Asiago SN archive, Benetti et al. 2004). In spite of the paucity of $\mathrm{Fe}$-group elements observed at early epochs, as discussed in Sect. 5, this nebular spectrum apparently shows all the expected iron features, suggesting that iron-group elements were prevalent in the inner layers. At late phases SN 2004dt, resembles SN 2002bo (Benetti et al. 2004), which is in fact another HVG SN, but it also shows some peculiarities that deserve further investigation, such as the $\mathrm{Fe}$ [III]/ Fe [II] line ratio, with the $\mathrm{Fe}[\mathrm{III}] \lambda \sim 4700 \AA$ and $\mathrm{Fe}[\mathrm{II}] \lambda \sim 5300 \AA$ features with similar peak intensity, and an intriguing $\mathrm{Fe}[\mathrm{II}] \lambda \sim 4400 \AA$ feature that is particularly strong. The late spectral evolution of SN 2004dt will be analyzed and discussed in more detail in a forthcoming paper.

\section{Spectral modeling}

The availability of early SNe Ia spectra, whose number is increasing thanks to observational efforts, such as the ones performed by the European Supernova Collaboration, shows that the high-velocity line features are quite common in $\mathrm{SNe} I \mathrm{Ia}$ 


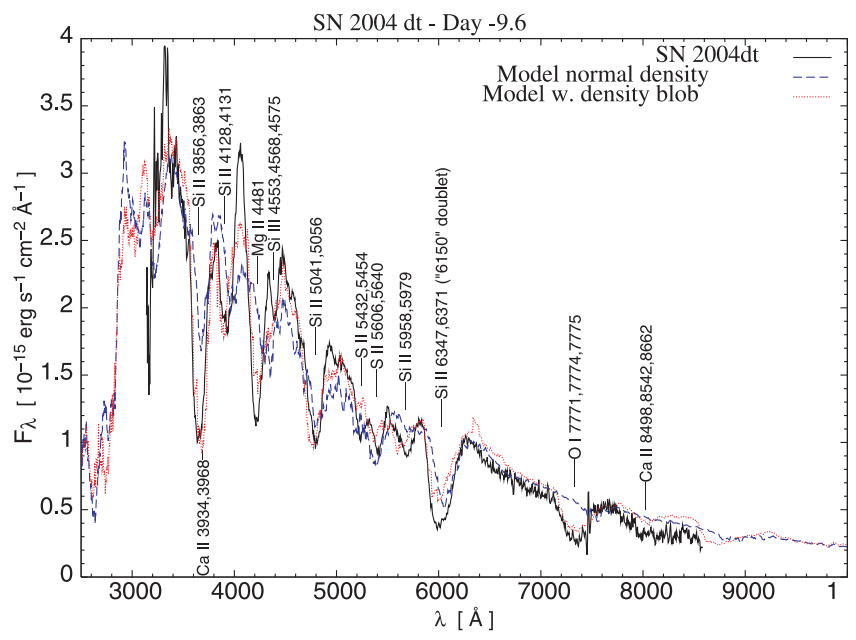

Fig. 14. Observed spectrum and corresponding model of SN 2004dt at day -9.6 using $E(B-V)=0.0, \mu=34.61, t_{\exp }=9.1 \mathrm{~d}$.

(Mazzali et al. 2005b; Benetti et al. 2005; Garavini et al. 2007), but most objects only show high-velocity components in some lines, preferentially in Si II and Ca II lines. SN 2004dt seems to be an extreme case. All lines of the early time spectra, except for sulfur, have prominent high-velocity absorptions. Even $\mathrm{O} I$ is strongly affected, which is very unusual. In order to find physical explanations of these aspects from a more theoretical point of view, spectral synthesis calculations were performed. We used a Monte Carlo code to calculate synthetic spectra. A detailed description of the code can be found in Abbott \& Lucy (1985), Mazzali \& Lucy (1993), and Lucy (1999). Another extension of the Abbott \& Lucy's (1985) code was developed for premaximum spectral analyses of SNe Ia (Ruiz-Lapuente 1992; Ruiz-Lapuente et al. 1992). Recently, the Lucy (1999) code was enhanced in order to derive the distribution of abundances in SNe Ia ejecta allowing detailed supernova tomography (Stehle 2004; Stehle et al. 2005).

Here the analysis is focused on an early spectrum and on another one near maximum light. The early epochs are bestsuited to examining the outer parts of the ejecta, hence the highvelocity components of the absorption lines. Since the SN ejecta have not yet expanded very much, the outer layers are still dense enough to produce significant absorptions.

Models for both epochs are initially based on the W7 density profile (Nomoto et al. 1984), which is taken as the standard departure model. The bolometric luminosity, the radius of the inner boundary ("photosphere"), and the abundances are determined iteratively to achieve the best match to the observed spectrum.

\subsection{Standard density profile}

The spectrum at day -9.6 is chosen for modeling the early epoch. As shown in Fig. 2, it is the earliest spectrum that covers a large wavelength interval, from the UV to the near IR. Input distance $(\mu=34.61)$ was taken from the Lyon-Meudon Extragalactic Database $^{7}$, other input parameters, like the time since explosion $\left(t_{\exp }=9.1 \mathrm{~d}\right)$ and reddening $\left(E(B-V)=0.00^{8}\right)$, were derived from the observations. The dashed line in Fig. 14 shows the bestfitting model. The derived luminosity is $\log _{10} L=42.87\left(\mathrm{erg} \mathrm{s}^{-1}\right)$,

\footnotetext{
7 http://cismbdm. univ-lyon $1 . \mathrm{fr} / \sim$ leda/

8 Due to the discrepancies in the observed reddening discussed in Sect. 3 and the absence of clearly detectable Na I, we assumed the reddening to be negligible.
}

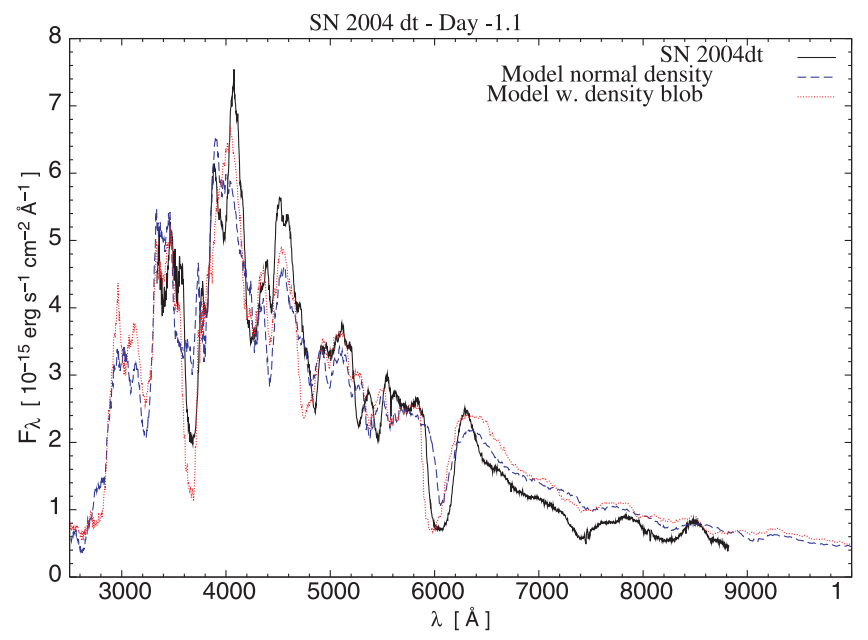

Fig. 15. Observed spectrum and corresponding model of SN 2004dt at day -1.1 using $E(B-V)=0.0, \mu=34.61, t_{\exp }=17.7 \mathrm{~d}$.

and the photospheric radius is located at $14500 \mathrm{~km} \mathrm{~s}^{-1}$. To account for the high-velocity components, three shells in the outer layers - at 18000, 20000 , and $23000 \mathrm{~km} \mathrm{~s}^{-1}$ - were introduced. These velocities correspond to the main regions where different lines were detected.

Two discrepancies between the model and the observations are evident: first, except for the S II W-feature, all synthetic lines are redder than the observed ones, or at least lack absorptions in their blue wings. Second, there is almost no Si II absorption at $5970 \AA$, and the Si III line at $4560 \AA$ is too strong, which suggests that the model temperature is too high. The Si III lines are formed near the photosphere, where the temperature is very high, while Si II lines are formed farther out, where the temperature is lower. Using a standard density profile leaves too little mass in regions above $\sim 15000 \mathrm{~km} \mathrm{~s}^{-1}$ to produce significant absorptions. Oxygen is singly ionized at low velocities, but the strong O I line at $7772 \AA$ is absent in the model, due to the low density at velocities $\sim 17000 \mathrm{~km} \mathrm{~s}^{-1}$, where its main observed absorption occurs. Only the S II W-feature ( $5630 \AA)$ can be reproduced fairly well. These lines are comparably weak and are formed near the photosphere. Therefore, they do not show significant high-velocity absorption.

The maximum light spectrum was assumed to have an epoch from explosion of $t_{\exp }=17.7 \mathrm{~d}$. The photospheric velocity had decreased to $9200 \mathrm{~km} \mathrm{~s}^{-1}$, so the model luminosity was determined to be $\log _{10} L=43.04\left(\mathrm{erg} \mathrm{s}^{-1}\right)$. Figure 15 shows that the original W7 model (dashed line) behaves as at the early time. The overall flux shape is reproduced well, but most lines are too weak compared with the observed spectrum. Again, the temperature near the model photosphere is high enough that $\mathrm{Si}, \mathrm{Mg}$, and Fe are doubly ionized. Therefore, the transitions of singly ionized species are too weak in the synthetic spectrum, seen best in Si II $\lambda 3860 \AA$, $4130 \AA$, and $6355 \AA$. Consequently, the Si III $\lambda 4560 \AA$ line is too strong compared with the observed one.

The two spectral synthesis models show that the deep, blue-line absorption cannot be reproduced by simply increasing the abundances, especially because HVFs affect not just a few lines but almost all lines - including O I $\lambda 7772 \AA$. Since more absorption at higher velocities is necessary and increasing the abundances in the outer regions is not sufficient, the other possibility is to increase the density. Plausible reasons for an enhanced density are discussed later. 


\subsection{Enhanced density profile}

To improve the models, the density at high velocities was increased in steps until the line absorptions could be reproduced both in depth and velocity. Furthermore, attention was paid to keeping the density profile smooth and similar to the original shape of the W7 profile. We also tried to keep the abundances at reasonable values, i.e. similar to the values that apply to other "normal" SNe Ia. The best match was achieved by adding $0.26 M_{\odot}$ above $16000 \mathrm{~km} \mathrm{~s}^{-1}$ in a spherically symmetric shell.

The dotted line in Fig. 14 shows the model, using the same input parameters and abundances as before, but with the higher density. The improvements compared to the model without a density enhancement are striking. The combined feature of Ca II, H\&K, and Si II $\lambda 3856 \AA$, as well as the Si II $\lambda 4130 \AA$ line, are reproduced very well, both in absorption depth and velocity. The Mg II $\lambda 4481 \AA$ line is somewhat too shallow, but now it is at the correct wavelength. Si III at $\lambda 4560 \AA$ is strongly reduced compared with the low-density model, while the Si II $\lambda 5045 \AA$ line is deeper and fits the observations very well. The S II absorption between $5090 \AA$ and $5660 \AA$ is slightly weaker, but it still is at the right place, and a significant absorption due to Si II $\lambda 5960 \AA$ is visible. Lower temperatures in the outer layers, in comparison with those near the photosphere, allow this line to be formed. The main Si feature, Si II at $\lambda 6350 \AA$, is still not deep enough, but the model shows the high-velocity component that is not present in the low-density model. The effect of an increased density can be seen nicely for O I $\lambda 7772 \AA$. This line was completely absent in the model without a density enhancement, but now the model matches the observed line profile almost perfectl

The model near maximum light shows a similar change when the density is increased in the same way as for the model at the early epoch. All lines are shifted bluewards. The Si II lines at $3856 \AA$ and $6350 \AA$ are comparable to the observations. Fe III at $4420 \AA$ comes in and dominates the feature observed at $\sim 4250 \AA$. The Si III complex at $4560 \AA$ is still a little bit too strong but represents the observed line much better. A fairly strong absorption near $4750 \AA$ is caused by Si II 5041, $5056 \AA$. In the model it appears to be formed at velocities that are too high. The same applies to the main Si II feature at $6350 \AA$, which is blue-shifted with respect to the observations. Even with an increased density, there is not enough absorbing mass to reach the depth of the O I $\lambda 7772 \AA$ line. Also the Ca II IR-triplet is underestimated in the model. A higher $\mathrm{Ca}$ abundance, however, would also increase the absorption depth of Ca II, H\&K, which is already too strong.

From the analysis using synthetic spectra, it can be concluded that SN 2004dt contains an unusually high Si abundance, since almost every prominent feature in the spectra is dominated by transitions of this element. This applies not only to the early epochs but also to the spectrum near maximum light, when usually $\mathrm{Si}$ high-velocity components tend to disappear. Even the deep feature near $3700 \AA$, which is normally Ca II, H\&K in most $\mathrm{SNe}$ Ia, is dominated by Si II transitions. On the other hand, only very little Fe-group elements are found in SN 2004dt. The outer layers $\left(14500 \mathrm{~km} \mathrm{~s}^{-1} \leq v \leq 20000 \mathrm{~km} \mathrm{~s}^{-1}\right)$ contain $0.5 \%$ by mass of Fe-group elements, and even in deeper layers between $9200 \mathrm{~km} \mathrm{~s}^{-1}$ and $14500 \mathrm{~km} \mathrm{~s}^{-1}$ only $12 \%$ can be found, where ${ }^{56} \mathrm{Ni}$ and ${ }^{56} \mathrm{Fe}$ begin to dominate in other SNe Ia.

The analysis of the observations has already shown the significant high-velocity absorptions in almost every line except for the S II and Si III lines. Modeling those spectra has confirmed that, and proved that models with classical density profiles do not contain enough material in their outer regions to reproduce the observations. Objects like SN 2004dt clearly show in the spectral features the effects of 3D structures in the expanding ejecta, and so, they are a very strong hint of aspherical explosion scenarios.

\section{Discussion and conclusions}

SN 2004dt clearly differs from most "normal" SNe Ia with respect to line velocities. The observed high-velocity absorption can be reproduced in synthetic spectra only when the density is increased significantly above $\approx 15000 \mathrm{~km} \mathrm{~s}^{-1}$ with respect to models that fit the spectra of less extreme HVG SNe (Stehle et al. 2005). Global enhancement may be a property of the explosion or the result of interaction with circumstellar material (CSM) (Gerardy et al. 2004; Mazzali et al. 2005a). In both cases, a spherically symmetric situation is unlikely: a density enhancement would require a mass $\approx 0.26 M_{\odot}$, while the added mass in the case of interaction would be even larger since the CSM would probably be dominated by elements such as $\mathrm{H}$ and/or $\mathrm{He}$. A possibility is that the outer ejecta are shaped by rising fingerlike structures produced in the burning and that these may interact with CSM. The ubiquitous presence of HVFs in the earliest spectra of SNe Ia (Mazzali et al. 2005b) was reproduced by 3D spectrum synthesis models assuming a limited number of blobs (6 to 12) of large angular size at $v \sim 18000-25000 \mathrm{~km} \mathrm{~s}^{-1}$ (Tanaka et al. 2006). If one such blob happened to cover the early-time photosphere of SN2004dt almost completely, the observed line profiles may be recovered, while the total mass of high-velocity material would not exceed $\sim 0.1 M_{\odot}$.

The idea of finger-like or blob structures can probably be extended to all HVG SNe. SN2004dt is a member of a group of SNe Ia with unusually high-velocity absorption lines, indicating ejecta moving $4000-5000 \mathrm{~km} \mathrm{~s}^{-1}$ faster than the velocities typically measured in most $\mathrm{SNe}$ Ia at the same phases (Leonard et al. 2005). Other $\mathrm{SNe}$ in this class include SN 1981B, SN 1983G, SN 1984A, SN 1989B, SN 1991M, SN 1995al, SN 1997bp, SN 2002bo，SN 2002bf, SN 2002dj, SN 2002er (see also Benetti et al. 2005), 2005cf (Pastorello et al. 2007; Garavini et al. 2007). In the case of SN 2002bf, the spectropolarimetric data of Leonard et al. (2005) show similarities with SN 2004dt.

From the analysis of the spectropolarimetry, Leonard et al. (2005) favor clumpiness of the outer layers. Clumpiness of the ejecta is also supported by Wang et al. (2007), who find that the greater the line polarization (i.e. clumpiness), the faster the decline and the less luminous the SN. Due to the clumpy structure of the ejecta, projection effects may alter the observed polarization, suggesting that the peak luminosity/decline rate relationship in the light curves of SNe Ia could be explained by the varying clumpiness of the ejecta, combined with the viewing angle. Remarkably, SN 2004dt deviates significantly from the correlation between the degree of polarization across the Si II $\lambda 6355 \AA$ line $\left(P_{\text {SiII }}\right)$ and the decline rate $\Delta m_{15}(B)$ found by Wang et al. (2007), confirming its peculiarity. The same authors suggest that projection effects may account for this deviation. Clumpiness has also been observed in the remnant of Tycho's SN (Badenes et al. 2005). Although based on just 1-D modeling of the ejecta, our own results point in the same direction.

It is still unclear what type of explosion is most suitable for producing intrusions of intermediate-mass elements moving at high velocities into (or above) outer layers of unburned $\mathrm{O}$ and C. Subsonic burning, the defining characteristic of deflagration models of the explosions, can reproduce such mixing, whereas detonation models are thought to burn the outer layers without producing clumps. A possible exception to the latter 
could be the model of Plewa et al. (2004), where the intrusion of high-velocity, intermediate-mass elements would come from the same rising plume that triggers a detonation in the outer layers (Kasen \& Plewa 2005). However, the presence of a single blob would be at odds with the presence of HVFs in all SNe Ia.

The combination of the spectroscopic and photometric observations of SN 2004dt with 1D modeling of the ejecta and of the corresponding spectra presented here strengthens the conclusions from independent spectropolarimetric analyses of this and similar SNe Ia. It equally stresses the need for realistic 3D models of the explosion to deal with current and future data, and also the importance of early observations, both spectroscopic and spectropolarimetric.

Acknowledgements. This work has been supported by the European Community's Human Potential Program under contract HPRN-CT-2002-00303, "The Physics of Type Ia Supernovae".

This work is based on observations collected at the $2.3 \mathrm{~m}$ telescope at the Siding Spring Observatory (SSO, Australia), at the $2.2 \mathrm{~m}$ telescope at the Centro Astronómico Hispano Alemán (CAHA, Spain), at the $3.6 \mathrm{~m}$ Telescopio Nazionale Galileo (TNG, La Palma, Canary Islands), at the $2.5 \mathrm{~m}$ Nordic Optical Telescope (NOT, La Palma, Canary Islands), and at the $1.8 \mathrm{~m}$ telescope at the Asiago Astrophysical Observatory (Italy). The SSO is operated by the Australian National University. The CAHA at Calar Alto is operated jointly by the MaxPlank Institut für Astronomie and the Instituto de Astrofísica de Andalucía (IAA-CSIC). The TNG is operated on the island of La Palma by the Centro Galileo Galilei of the Istituto Nazionale di Astrofisica (INAF), in the Spanish Observatorio del Roque de Los Muchachos of the Instituto de Astrofísica de Canarias. The NOT is operated on the island of La Palma jointly by Denmark, Finland, Iceland, Norway, and Sweden, in the Spanish Observatorio del Roque de Los Muchachos of the Instituto de Astrofísica de Canarias. The Astrophysical Observatory of Asiago is operated by the Padua Observatory (member of the National Institute for Astrophysics INAF) and by the Astronomy Department of the University of Padua.

We would like to thank J. Näränen, K. Muinonen, X. Wang, M. Weidinger, R. Karjalainen, and J. Telting for observing SN 2004dt at NOT. We are grateful to all the other observers and to the staff at the various observatories for efficiently scheduling and carrying out our requests and to the observers who had to give up part of their observing time so that SN 2004dt could be observed.

This research made use of the NASA/IPAC Extragalactic Database (NED), which is operated by the Jet Propulsion Laboratory, California Institute of Technology, under contract with the National Aeronautics and Space Administration. We have also made use of the Lyon-Meudon Extragalactic Database (LEDA), supplied by the LEDA team at the Centre de Recherche Astronomique de Lyon, Observatoire de Lyon.

\section{References}

Abbott, D. C., \& Lucy, L. B. 1985, ApJ, 288, 679

Altavilla, G., Fiorentino, G., Marconi, M., et al. 2004, MNRAS, 349, 1344

Badenes, C., Borkowski, K. J., Hughes, J. P., Hwang, U., \& Bravo, E. 2006, ApJ, 645,1373

Benetti, S., Meikle, P., Stehle, M., et al. 2004, MNRAS, 348, 261

Benetti, S., Cappellaro, E., Turatto, M., et al. 2005, ApJ, 623, 1011

Branch, D., Garnavich, P., Matheson, T., et al. 2003, AJ, 126, 1489

Burstein, D., \& Heiles, C. 1982, AJ, 87, 1165

Cappellaro, E., Patat, F., Mazzali, P. A., et al. 2001, ApJ, 549, 215

Cristiani, S., Cappellaro, E., Turatto, M., et al. 1992, A\&A, 259, 63

Filippenko, A. V., Li, W. D., Treffers, R. R., \& Modjaz, M. 2001, ASPC, 246, 121

Gamezo, V. N., Khokhlov, A. M., Oran, E. S., Chtchelkanova, A. Y., \& Rosenberg, R. O. 2003, Science, 299, 77
Garavini, G., Nobili, S., Taubenberger, S., et al. 2007, A\&A, 471, 527

Gerardy, C. L., Höflich, P., Fesen, R. A., et al. 2004, ApJ, 607, 391

Gómez, G., \& López, R. 1998, AJ, 115, 1096

Hillebrandt, W., \& Niemeyer, J. C. 2000, ARA\&A, 38, 191

Horne, K. 1986, PASP, 98, 609

Kasen, D., \& Plewa, T. 2005, ApJ, 622, 41

Khoklov, A. 1991, A\&A, 245, 114

Kirshner, R. P., Jeffery, D. J., Leibundgut, B., et al. 1993, ApJ, 415, 589

Kotak, R., Meikle, W. P. S., Pignata, G., et al. 2005, A\&A, 436, 1021

Kuchner, M. J., Kirshner, R. P., Pinto, P. A., \& Leibundgut, B. 1994, ApJ, 426, L89

Lentz, E. J., Baron, E., Branch, D., et al. 2000, ApJ, 530, 966

Leonard, D. C., Li, W., Filippenko, A. V., Foley, R. J., \& Chornock, R. 2005, ApJ, 632, 450

Lira, P. 1995, Masters thesis, Univ. Chile

Livne, E. 1999, ApJ, 527, L97

Lucy, L. B. 1999, A\&A, 345, 211

Lundqvist, P., Sollerman, J., Leibundgut, B., et al. 2003 in From Twilight to Highlight: The Physics of Supernovae: Proceedings of the ESO/MPA/MPE Workshop Held at Garching, Germany, 29-31 July 2002, ESO Astrophysics Symposia, ed. W. Hillebrandt \& B. Leibundgut (Springer-Verlag), 309

Marsh, T. 1989, PASP, 101, 1032

Maxted, P. F. L., Marsh, T. R., \& North R. C. 2000, MNRAS, 317, L41

Mazzali, P. A. 2001, MNRAS, 321, 341

Mazzali, P. A., \& Lucy, L. B. 1993, A\&A, 279, 447

Mazzali, P. A., Benetti, S., Stehle, M., et al. 2005a, MNRAS, 357, 200

Mazzali, P. A., Benetti, S., Altavilla, G., et al. 2005b, ApJ, 623, L37

Mazzali, P. A., Röpke, F. K., Benetti, S., \& Hillebrandt, W. 2007, Science, 315, 825

Moore, M., \& Li, W. 2004, IAUC 8386

Napiwotzki, R., Karl, C., Nelemans, G., et al. 2004, RMxA\&A, 20, 113

Nomoto, K., Thielemann, F.-K., \& Yokoi, K. 1984, ApJ, 286, 644

Nugent, P., Phillips, M., Baron, E., et al. 1995, ApJ, 455, 147

Nugent, P., Baron, E., Branch, D., Fisher, A., \& Hauschildt, P. H. 1997, ApJ, 485,812

Pastorello, A., Taubenberger, S., Elias-Rosa, N., et al. 2007, MNRAS, 376, 1301

Patat, F., Benetti, S., Cappellaro, E., et al. 1996, MNRAS, 278, 111

Patat, F., Pignata, G., \& Benetti, S. 2004, IAUC 8387

Perlmutter, S., Aldering, G., Goldhaber, G., et al. 1999, ApJ, 517, 565

Pignata, G., Patat, F., Benetti, S., et al. 2004, MNRAS, 355, 178

Pignata, G., Benetti, S., Buson, L., et al. 2005, in Supernovae as Cosmological Lighthouses, ed. M. Turatto, S. Benetti, L. Zampieri, \& W. Shea, 1604-2004 (San Francisco: ASP), ASP Conf. Ser., 342, 266

Plewa, T., Calder, A. C., \& Lamb, D. Q. 2004, ApJ, 612, L37

Pskovskii, Y. P. 1969, SvA, 12, 750

Reinecke, M., Hillebrandt, W., \& Niemeyer, J. C. 2002, A\&A, 391, 1167

Riess, A., Riess, A. G., Filippenko, Alexei, V., et al. 1998, AJ, 116, 1009

Röpke, F. K., Woosley, S. E., \& Hillebrandt, W. 2007, ApJ, 660, 1344

Ruiz-Lapuente, P. 1992, Ph.D. Thesis, Univ. of Barcelona

Ruiz-Lapuente, P., Cappellaro, E., Turatto, M., et al. 1992, ApJ, 387, L33

Ruiz-Lapuente, P., Comeron, F., Méndez, J., et al. 2004, Nature, 431, 1069

Salvo, M. E., Cappellaro, E., Mazzali, P. A., et al. M. 2001, MNRAS, 321, 254

Salvo, M. E., Schmidt, B., \& Wood, P. 2004, IAUC, 8387

Schlegel, D. J., Finkbeiner, D. P., \& Davis, M. 1998, ApJ, 500, 525

Schmidt, B. P., Kirshner, R. P., Leibundgut, B., et al. 1994, ApJ, 434, 19

Stanishev, V., et al. 2006, A\&A, submitted

Stehle, M. 2004, Ph.D. Thesis, Ludwig-Maximillians-Univ.

Stehle, M., Mazzali, P. A., Benetti, S., \& Hillebrandt, W. 2005, MNRAS, 360, 1231

Tanaka, M., Mazzali, P. A., Maeda, K., \& Nomoto, K. 2006, ApJ, 645, 470

Thielemann, F.-K., Nomoto, K., \& Yokoi, K. 1986, A\&A, 158, 17

Theureau, G., Bottinelli, L., Coudreau-Durand, N., et al. 1998, A\&AS, 130, 333

Thomas, R. C., Aldering, G., Antilogus, P., et al. 2007, ApJ, 654, 53

Wang, L., Baade, D., Höflich, P., et al. 2006, ApJ, 653, 490

Wang, L., Baade, D., \& Patat, F. 2007, Science, 315, 212 